\title{
ProActive Service Entity Framework: Improving Service Selection Chances within Large Senior Professional Virtual Community Scenario
}

\author{
Tiago Cardoso and Luis M. Camarinha-Matos \\ Faculty of Science and Technology, Universidade Nova de Lisboa, \\ Quinta da Torre, Caparica, Portugal \\ \{tomfc, cam\}@uninova.pt
}

\begin{abstract}
Within a Collaborative Business Ecosystem context, as the network evolves, the competition between members increases and, as a consequence, members face the challenge to improve the chances their services have to be selected. On the other hand, the distance between Business Services and "Computational Services" is still a bottleneck for the automation of service provision. This paper extends the Pro-Active Service Entity Framework, proposing a mechanism to improve the service selection chances, based on the refinement of a Quality of Service concept for this context, and introduces a new class of actors for the framework - the intermediaries, needed to shorten the distance between Business and Computational perspectives.
\end{abstract}

Keywords: Business Services, Web-Services, Professional Virtual Community.

\section{Introduction}

Technological Services, such as Web Services, and Business Services (BSs), have gained the attention of the research community in the last decades, especially in the computer science area. The creation of the Web Services (WSs) approach, in a first stage, and the appearance of the Service Oriented Architectures, in a later stage, constitute nowadays the most commonly accepted and adopted mechanisms to support the development of ICT systems that support BSs. Nevertheless, although the advances in this technological perspective have significantly changed the way ICT systems support BSs there still is a gap between the way business people see the services they, or their enterprises, are willing to provide to customers or clients and the counterpart provided by the technological persons that develop the underlying ICT systems to support such provision. In other words, the business perspective of the services that either professionals or enterprises provide to clients is related with the client satisfaction, the service value or the resource management and the involved processes in such service provision. On the other hand, the ICT people's perspective frequently focuses on interoperability, remote procedure calling or web-service 
composition. The same gap exists within a Collaborative Business Ecosystem, where the members have a distinct way of seeing the services they are willing to provide to the community and the adopted web-services technological approach.

The passiveness of web services, in the sense they stay still waiting for the client's initiative, and the inexistence of aggregation between distinct Web-Services from the same provider are considered the major problems of current approaches, according to [1], where the authors propose a solution based on Multi-Agent Systems to target the passiveness problem, extending the Service Entity concept, first proposed in [2], in order to tackle the aggregation of distinct services provided by the same provider. Later, in [3] a first proposal is made for the Pro-Active Service Entity Framework (PASEF), extended in this paper, in order to create a mapping between Business and Software perspectives of service. In fact, PASEF constitutes a form of a Service Park, as identified in [4] bringing elements from the Multi-Agent Systems to the Collaborative Business Ecosystems, as foreseen in [5].

In this paper, the typical usage of PASEF within a Professional Virtual Community (PVC) of Senior Professionals is presented and the introduction of an additional class of actors is made - the PVC Intermediary. This actor has the role of bridging between the Senior PVC members and the clients, forming a team of value co-creation. One of the major tasks for this co-creation is the transformation of the business needs specified by clients into the identification and composition of the needed business services provided by the members of the PVC - the Senior Professionals. Finally, a mechanism for the calculation of Quality of Service is also proposed to support this task, especially useful in PVCs with a large number of members. This mechanism is inspired in [6], where bid evaluation considers client satisfaction, and [7], where bid arrival patterns are studied.

\section{Contribution to Technological Innovation for Sustainability}

Demographic evolution along with medical improvements that lead to longer average life expectation are two factors that compromise society's Sustainability. In other words, the number of working persons related to the number of retired persons are one of the major factors contributing for the world's Sustainability problems nowadays, as explained in [8]. Nevertheless, current trends point out that more people want to keep their active life after retirement and organizations are starting to support such "after retirement activity".

The contribution of this paper to Technological Innovation for Sustainability is aligned with these trends, proposing mechanisms to make Business and Software perspectives come closer within a senior PVC context. This proposal is based on the concept of value co-creation, evolving PVC Clients, the Senior Professionals and PVC Intermediaries, and based in a new mechanism proposed to assess Quality of Service from the Senior Professionals. 


\section{PASEF Proposal}

The ProActive Service Entity Framework is intended to provide a solution within a competitive Collaborative Networked Business Ecosystem, particularly in a PVC, for the members of these communities to benefit from a pro-active representation of the Business Services they are willing to provide, as well as for clients to benefit from a wide range of potential Business Service providers.

The PASEF illustrating scenario is a Senior Coaching Association (SCA), a not for profit organization, dedicated to Senior Professionals that intend to continue their professional life after retirement, helping economic development through consultancy / coaching services, based on their professional life experience. This choice was made given the challenging demographic sustainability, as described in [8].

The framework is based on the assumption of the existence of a third class of actors, other than the PVC members and clients - the PVC Intermediary - with the role of facilitating the interface between the seniors and the business clients, namely through identification and characterization of business opportunities, as well as creation of Business Process Models based on the high-level needs specified by clients.

PASEF is composed of two complementary systems:

- Pro-Active Service Entity (PSE) - which aim to represent in a pro-active manner the Business Services a PVC member is willing to provide.

- $\quad$ PVC-Portal - a kind of PVC management system and virtual collaboration space that interacts with Clients who post their needs in high-level specifications and also the PVC Intermediaries and the PSEs.

The typical PASEF usage is divided in three stages: 1 - the PVC Member Registration stage, 2 - the Business Opportunity specification stage, and 3 - the Business Process Execution stage.

In the PVC Member Registration stage, each community member creates his / her PSE in the Portal and configures specifying the Business Services he / she is willing to provide, as well as the general availability and the
Name: Pedro; Email: pedro@ @sca.org, MAX BOs: Not defined MAX Simultaneous BOs: 5, BID Posting Policy: Auto BO Check rate: Week, Availability: Mon / Wed / Fri - (9:00 - 12:00)

(\#, name, category, description, input info, result)

(1, "Live Marketing Strategy Definition", "Marketing Consultancy", "Marketing Consultancy with live meetings", "1st meeting date/time proposal", "PDF Report")

(2, "Remote Marketing Strategy Definition", "Marketing Consultancy", "marketing consultancy withOUT live meetings", "documentation ZIP", "PDF Report")

(3, "Marketing Results Monitoring", "Marketing Consultancy", "monitor marketing initiatives", "', "PDF Report")

Text Box 1. PSE Configuration example

PSE pro-activeness properties. Text Box 1 shows an example of a Senior that is willing to provide 3 consultancy services from the Marketing Consultancy Business Service Category. The first service, for example, is "Live Marketing Strategy Design", corresponding to a service that will be provided in some iterations and the input 
information is the proposal for the first iteration date and time. The final result of this service is a PDF Report, corresponding to the Marketing Strategy.

The registration stage ends when the PSE is launched and starts looking for Business Opportunities where the services it represents are needed. After this stage, the PASEF manages a pool of services with pro-active behavior, in whose collaboration space clients can post their business needs and wait for the best proposals, based on an assessment of the Quality of Service made by PASEF, and other possible attributes, as explained bellow. In this Business Opportunity specification stage, the typical sequence of actions is summarized as follows:

$1^{\text {st }}-$ Clients make high-level specifications of their needs - the Business Opportunities. Textbox 2 shows a simplified illustration of a need specification.

$2^{\text {nd }}-$ PVC Intermediaries, in collaboration with the clients, transform such high-level specifications into abstract Business Process Models (absBPM), composed of Business Services from the PASEF pool. After the

BO_ID - 2010-02-32

Company - FreshAir

Contact Person - José

Need Description:

Marketing policy coaching

Text Box 2. Client Need commitment of the Client with this absBPM, this step is concluded by posting a Call for Proposals (CfP), corresponding to each Business Service included in the absBPM, in a blackboard like interface within PASEF. Text box 3 shows a simplified CfP example from the same illustrative scenario, where the PVC Intermediary and the client decided that the needed absBPM for a Marketing Policy Coaching is the sequence of the Business Services: Marketing Strategy Definition, Marketing Plan Execution and Marketing Results Monitoring, as represented in Figure 1.

BO_ID - 2010-02-32

BID / Proposal Acceptance period - 5/2 - 15/2

Workflow Definition

[Order, Category ID, BS ID, name, input parameters, output]

[1, 77, 234, "Live Marketing Strategy Definition", [Meeting Date Proposal(Date/Time), documentation (ZIP)], [Report (PDF/Doc)]]

[2, 77, 456, "Marketing Plan Execution", [Marketing Plan (PDF/DOC)], [Final report (PDF/Doc)]]

[3, 77, 123, "Marketing Results Monitoring", [], [Final report (PDF/Doc)]]

Text Box 3. Client Need

\begin{tabular}{|c|c|c|}
\hline $\begin{array}{c}\text { Marketing Strategy } \\
\text { Definition }\end{array}$ & $\begin{array}{l}\text { Marketing Plan } \\
\text { Execution }\end{array}$ & \multirow{5}{*}{$\begin{array}{l}\text { BID (\#, BO_ID, [Srv_ID, DTime, ...], Senior_ID }) \\
\operatorname{BID}\left(1,2010 \_2 \_32,[1,1 \text { week }], 234\right) \\
\operatorname{BID}\left(2,2010 \_2 \_32,[2,2 \text { week }], 234\right) \\
\operatorname{BID}\left(3,2010 \_2 \_32,[(1,1 \text { week }),(2,1 \text { week })],\right. \\
234) \\
\text { BID }\left(4,2010 \_2 \_32,[2,5 / 4 \text { week }], 445\right) \\
\quad \text { Text Box 4. Illustrative Proposals }\end{array}$} \\
\hline & $\downarrow$ & \\
\hline & Marketing Results & \\
\hline & Monitoring & \\
\hline \multicolumn{2}{|c|}{ Fig. 1. Illustrative Workflow } & \\
\hline
\end{tabular}

$3^{\text {rd }}-$ All PSEs look up for a suitable CfP, in a pre-defined frequency rate, to find opportunities where one or more of the services they represent are in need. Whenever this search has a successful result, they prepare / submit a proposal for the provision of such Business Service. Text box 4 shows an example of the posted proposals for the illustrative scenario. This is a special case where one PSE (ID: 234) represents two of the needed Business Services for a specific Business Opportunity. As a result 
of this fact, the PSE prepares three proposals: one for each BS independently and one for the case of the provision of the two services.

$4^{\text {th }}$ - After a pre-defined time period or the arrival of a specific number of proposals, the PVC Intermediary closes the CfP and selects the best ones for each case, based on Quality of Service and other criteria, completing the executable BPM (eBPM). This step is concluded with another commitment of the Client, this time with this eBPM, corresponding to an agreement with the service providers' selection.

At a high level, this process can be seen as an instantiation of the well known contract-net protocol of the multi-agent systems area [9]. There are, however, several differences resulting from the combination of pro-activeness with the service notion.

Finally, after the eBPM is ready, with all the Business Services identified, as well as the Service Providers selected, the Business Process Execution stage starts when the Client launches the eBPM execution. From that point on, PASEF is responsible to call the included Business Services through the corresponding PSEs at the right time. The Service Providers are then informed to start the provision of their Business Services, eventually receiving needed input information in order to produce the corresponding output results.

One of the major objectives of this proposal is to bridge between the Business Services that PVC members provide and the technological services, Web-Services or other mechanisms, used to support this provision. In this context a Business Service is considered as an independent executing entity whose internal details, like task workflow or local data, are not taken into account, hence a BS is treated as a black box, although it must have an execution state and eventually input data and output results specification. Five distinct execution states are considered in a BS lifecycle:

1. Launch - the BS is ready to be launched by PASEF, when the time within the corresponding eBPM comes.

2. Ready - the PSE is ready to ask the community member it represents to start / continue the service provision / execution. This state happens in two situations: at the beginning of the BS lifecycle, when the service is launched, and whenever something the BS is waiting for becomes available - for example some documentation provided by another BS.

3. Run - the community member is executing the service.

4. Wait - the community member waits for some information from another community member, for example.

5. Complete - the BS gets complete and the results are eventually sent to PASEF, through the PSE, so they can be used as input parameters for subsequent BSs from the corresponding eBPM.

PASEF provides a toolbox for the PVC actors to trigger execution state transitions on such BSs, as well as to support any interaction between the PSEs of distinct BSs. Figure 2 represents the relation between the BS and PASEF Web-Services, for the case of a single BS execution. This execution state machine is repeated for each BS included in the eBPM. 


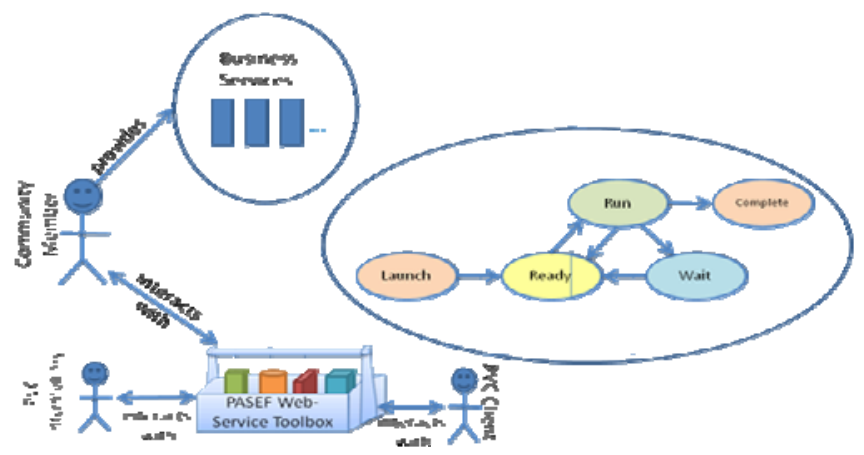

Fig. 2. PASEF bridge between Business and Technological Services

It is interesting to notice the similarity between the 5 identified BS states and the states a task can have in real-time-systems. Although the abstraction level is distinct, they both are execution entities, take their time executing and depend / interact with the surrounding environment. In the case of tasks from a real-time-system, the work is done by processors and the state-transitions-machinery is carried out by a Real-TimeKernel. In the Case of these Business Services, the work is done by the PVC members and the state-transition-machinery is carried out through the PASEF toolbox.

\subsection{PVC-Park and Pro-active Service Entity Web-Service functionality}

The Web-Services provided by both PASEF PSEs and Portal are represented in Figure 3 and detailed in table 1 and 2.

\begin{tabular}{|c|c|c|c|c|c|}
\hline $\begin{array}{l}\text { Rezi } \\
\text { Mane }\end{array}$ & $\underbrace{\text { PSE }}_{\substack{\text { Btion } \\
\text { Ment }}}$ & Fig. 3. PVC & $\begin{array}{c}\text { PV } \\
\begin{array}{c}\text { Client } \\
\text { interface }\end{array} \\
\end{array}$ & $\sum_{\text {Manoment }}^{\text {C-Portal }}$ & ent \\
\hline \multicolumn{3}{|c|}{ Table 1. PASEF PSE WS Functionality } & \multicolumn{3}{|c|}{ Table 2. PASEF Portal WS Functionality } \\
\hline WebService & Functionality & WS Func.Client & WebService & Functionality & WS Func.Client \\
\hline \multirow{2}{*}{$\begin{array}{c}\text { Registration } \\
\text { Managmnt }\end{array}$} & \multirow[b]{2}{*}{ Service Registration } & \multirow{2}{*}{ Member } & Client Interface & New BO / New Need & Clnt \\
\hline & & & \multirow{3}{*}{ Bo Managmnt } & BO Search & PSE \\
\hline \multirow[b]{2}{*}{ BID Managmnt } & BID Commit & Member & & BID Submission & PSE \\
\hline & \begin{tabular}{|l|} 
BID Acceptance \\
Notification
\end{tabular} & PVC Portal & & \begin{tabular}{|l|} 
BID Submit \\
Acceptance Result
\end{tabular} & PVC_Interm. \\
\hline $\begin{array}{c}\text { Business Service } \\
\text { Execution }\end{array}$ & \begin{tabular}{|l|} 
Business Service Start \\
Business Service Cancel \\
\end{tabular} & $\begin{array}{l}\text { PVC Portal } \\
\text { PVC Portal }\end{array}$ & \multirow{2}{*}{$\begin{array}{l}\text { BPM Managmnt } \\
\text { (Edition stage) }\end{array}$} & $\begin{array}{l}\text { BPM Specification } \\
\text { Submission }\end{array}$ & PVC_Interm. \\
\hline \multirow{6}{*}{\multicolumn{3}{|c|}{$\begin{array}{l}\text { These Web-Services act as a toolbox for } \\
\text { he three actors that interact with } \\
\text { ASEF to be able to trigger the state }\end{array}$}} & & BPM Commit & Clnt \\
\hline & & & \multirow{5}{*}{$\begin{array}{l}\text { BPM Managmnt } \\
\text { (Execution stage) }\end{array}$} & eBPM Launch Execution & Clnt \\
\hline & & & & BPM Cancel Execution & Clnt \\
\hline & & & & BS Wait for Input & PSE \\
\hline & & & & $\begin{array}{l}\text { BS End Notification / } \\
\text { [result submition] }\end{array}$ & PSE \\
\hline & & & & BPM Execution Status & pVC Portal \\
\hline
\end{tabular}


order to make the bridge between Business Services from PVC members and this technological service set, in this case Web-Services.

\subsection{PASEF Quality of Service}

The mechanism used within PASEF to calculate the Quality of Service data is based on tracking all the service provision processes. Along time, PASEF stores information concerning all services, all service providers and all service provision instances. Based upon this information a configurable mechanism is provided for the PVC Intermediary, along with the Clients, to define the best way to classify and sort service provision proposals in order to make the best selection in all Business Opportunities. This mechanism is based in two concepts:

$>$ QoSCharacteristic - Some property concerning the service that may be measured and compared between distinct services. This is the atomic mechanism information that is defined in terms of a name, the information that may be measured, and the unit used in that measurement. Two QoS Characteristic categories were identified concerning the:

1. Service Provider - historic statistical information from the provider, e.g.:

- N_BOs - number of BOs where the PVC member was involved,

- BID_Success_Rate - BID Acceptance / Success Rate

- Satisfaction - customer satisfaction level, graded in an after-service form,

- OnTimeDeliverRate - \% of on time delivery instances,

- DelayOnDeliveryAverage - Average time of delay,

- ...

2. Service Provision - information from the Service Provision Proposal itself, specific to each proposal, e.g.:

- DTime - proposed result delivery time,

- ...

QoS Criteria: the definition of the QoS Criteria consists in five tasks that have to be carried out by the PVC Intermediary with the help of the Client:

1. Selection of the relevant QoS

Characteristics for the specific Business Opportunity, e.g. N_BOs , DTime, Satisfaction.

2. Definition / Selection of the evaluation schema, e.g., three classification levels: level 1(best), level 2 and level 3 (worst).

3. Definition of how the selected QoS Characteristics "fit" into

QoSCharacteristics Category weigh: Criteria schema: Level 1 / Level 2 / Leve 3. Restrictions:

-Level 1 level mandatory for OnTimeDeliver -DTime $<15$ and DelayOnDeliveryAverage $<4$ -Satisfaction not equal Level 3

Final Grading Formula:

$$
\begin{gathered}
\text { OnTimeDeliver * Satisfaction } \\
\text { N_Consortium_Members*N_BOs*(15 - DTime) }
\end{gathered}
$$

Text Box 5. Sample QoS specification the specified schema, e.g.

- N_BOs - Level 3 (values < 5); Level 1 (values > 15); Level 2 (otherwise)

- DTime - Level 3 (values < 5); Level 1 (values > 15); Level 2 (otherwise)

- Satisfaction - Level 3 (values < 5); Level 1 (values $>8$ ); Level 2 (otherwise) 
4. Definition of restrictions, e.g. no proposals should be considered from providers with Level 3 classification concerning NBOs.

5. Definition of the overall rating formula for a provision proposal. This formula is created using the QoS Characteristics selected in 1 and is then used to classify all proposals to sort them in order to make the best selection.

\section{Concluding Remarks}

The extension of the PASEF proposal presented in this paper targets a better contribution for shortening the conceptual distance between business and software perspectives to the service concept. In fact, the adoption of a team built up by clients, providers and an intermediary in value co-creation brings to PASEF the missing roles a middle entity has to perform.

The application of this framework to the challenging area of active ageing is both actual and vital in the economic perspective, given the continuous increase in aged population and the needed demographic sustainability, among other factors.

Finally, with the definition of the mechanism for the assessment of Quality of Services, the PVC members see the excellence of their services rewarded.

As future work, the PVC Intermediary role has to be extended to the execution of eBPMs stage, in order to follow and track the evolution of the evolved processes and, eventually react to deviations on the pre-defined model. As a result, the corresponding ICT evolution will originate another extension of PASEF.

\section{References}

[1] Cardoso, T., Camarinha-Matos, L.M.: Pro-active asset entities in collaborative networks. In: Camarinha-Matos, L.M., Pereira, P., Ribeiro, L. (eds.) DoCEIS 2010. IFIP AICT, vol. 314, pp. 93-102. Springer, Heidelberg (2010)

[2] Franco, R.D., Bas, Á.O., Esteban, F.L.: Modeling extended manufacturing processes with service oriented entities. Service Business 3, 31-50 (2008)

[3] Cardoso, T., Camarinha-Matos, L.M.: Pro-Active Service Entity Framework - Towards better mapping between Business and Software. In: Camarinha-Matos, L.M., Boucher, X., Afsarmanesh, H. (eds.) PRO-VE 2010. IFIP AICT, vol. 336, pp. 451-460. Springer, Heidelberg (2010)

[4] Petrie, C., Bussler, C.: The Myth of Open Web-Services - The Rise of the Service Parks. IEEE Internet Computing (2008)

[5] Camarinha-Matos, L.M.: Multi-Agent Systems in Virtual Enterprises. In: Proceedings of the International Conference on AI, Simulation and Planning in High Autonomy Systems (AIS 2002), SCS publication (2002)

[6] Pang, N., Shi, Y., Ling, Y.: Bid Evaluation Method of Construction Project Based on Relevance and Relative Closeness. In: Proceedings of the International Conference on Information Management, Innovation Management and Industrial Engineering (2008) 
[7] Xing-jian, X., Shi, A., Bo, W.: Comparison Study of Duration and Bid Arrivals in eBay Auctions across Different Websites. In: Proceedings of the International Conference on Management Science \& Engineering (2008)

[8] Camarinha-Matos, L.M., Afsarmanesh, H.: Active Ageing Roadmap - A Collaborative Networks Contribution to Demographic Sustainability. In: Camarinha-Matos, L.M., Boucher, X., Afsarmanesh, H. (eds.) PRO-VE 2010. IFIP AICT, vol. 336, pp. 46-59. Springer, Heidelberg (2010)

[9] Smith, R.G.: The Contract Net Protocol: High-level Communication and Control in a Distributed Problem. IEEE Transactions on Computers, 1104-1113 (1980) 